\title{
Acid, alkali and heat adaptation studies of Bacilli isolated from alkali cleaning solution in a Dairy Plant
}

\author{
Amitha Thomas ${ }^{1}$ Prasad $\mathrm{V}^{2}$ \\ Department of Dairy Science, Kerala Veterinary and Animal Sciences University, India.
}

\begin{abstract}
In this study bacteria were isolated from alkali cleaning solution used for cleaning of pasteurization in a Dairy Plant. The thermoduric bacteria from these samples were isolated after laboratory pasteurization in nutrient agar. The isolated samples were identified as genus Bacillus by morphology, cultural characters and biochemical reactions. The isolates were subjected to various $\mathrm{pH}$ treatments ranging from 4.5 to 10.5 and temperature range of 60 to $80^{\circ}$. The growth of organisms estimated by enumeration of organisms at different times and the growth rate of each isolates at each $\mathrm{pH}$ values were calculated and plotted as a function of time.
\end{abstract}

Key Words: acid adaptation, alkali adaptation, heat adaptation, Thermoduric Bacilli

\section{Introduction}

The $\mathrm{pH}$ environment of the milk contact surfaces fluctuates between acidic (4) and alkaline (9) values during Cleaning In Place (CIP) procedures used to clean the dairy processing equipment (Alvarez et al., 1998). The temperature of the cleaning procedure usually ranges from $60-70^{\circ} \mathrm{C}$. Alkaline cleaners are those that have a $\mathrm{pH}$ higher than 7. They are commonly used in the dairy industry because they saponify fat and convert the fat to soap and can be removed with water. These are usually consist of sodium hydroxide (caustic soda) potassium hydroxide (caustic potash), sodium carbonate (soda ash), and sodium silicates. The most common acids found in dairy cleaning are the inorganic acids, such as phosphoric acid and nitric acid, and the organic acids, such as citric acid. The organisms surviving the acid and alkaline $\mathrm{pH}$ conditions will cause subsequent contamination of the pasteurized milk. Hence a study was conducted to isolate the organisms present in the alkaline cleaning solution to detect out their alkaline, acidic and heat adaptability.

\subsection{Alkaline Adaptation Of Isolates}

\section{Materials And Methods}

\subsubsection{Preparation of samples}

The isolated thermoduric bacilli were used for this study. The bacteria were grown overnight in nutrient broth $(\mathrm{pH} 7.4)$ at $37^{\circ} \mathrm{C}$ in an incubator. For alkaline adaptation studies $\mathrm{pH}$ of nutrient broth were adjusted with $1 \mathrm{~N} \mathrm{NaOH}$ to 8.5, 9.5 and 10.5. The tubes were inoculated with one per cent of the above prepared overnight broth culture. These tubes containing $\mathrm{pH}$ adjusted broth culture were used for alkaline adaptation studies.

\subsubsection{Enumeration of Alkaline adapted cells}

The enumeration (Mortan 2001) of the prepared samples were carried out at $0,15,30,45$ and 60 minutes of incubation at room temperature. The growth rate (cfu) of each isolate at each $\mathrm{pH}$ value was calculated and plotted as a function of time ( Lindsaya et al., 2000).

\subsection{Acid Adptation}

\subsubsection{Preparation of samples}

The isolated thermoduric bacilli were used for this study. The bacteria were grown overnight in nutrient broth $(\mathrm{pH} 7.4)$ at $37^{\circ} \mathrm{C}$ in an incubator. For acid adaptation studies $\mathrm{pH}$ of nutrient broth were adjusted with $1 \mathrm{~N}$ Hydrochloric acid $(\mathrm{HCl})$ to $4.9,5.5$ and 6.3. The tubes were inoculated with one per cent of the above prepared overnight broth culture. These tubes containing $\mathrm{pH}$ adjusted broth culture were used for acid adaptation studies.

\subsubsection{Enumeration of acid adapted cells}

Enumeration (Mortan 2001) of the prepared samples were carried out at 0,15, 30, 45 and 60 minutes of incubation at room temperature. The growth rate (cfu) of each isolate at each $\mathrm{pH}$ value was calculated and plotted as a function of time ( Lindsaya et al., 2000).

\subsection{Heat Adaptation}

The isolated thermoduric bacilli were used for this study. The bacteria were grown overnight in nutrient broth $(\mathrm{pH} 7.4)$ at $37 \mathrm{C}$ in an incubator. For heat adaptation studies the overnight broth cultures were inoculated into nutrient broth at $1 \%$ level and subjected to different heat treatments $65^{\circ} \mathrm{C}, 70^{\circ} \mathrm{C}$ and $80^{\circ} \mathrm{C}$. Enumeration 
(Mortan 2001) of the prepared samples were carried out at $0,15,30,45$ and 60 minutes of incubation. The growth rate (cfu) of each isolate at each $\mathrm{pH}$ value was calculated and plotted as a function of time (Lindsaya et al., 2000).

\section{Results}

The growth curve of the isolated organisms at three different alkaline $\mathrm{pH}$ is given in the Figure 1. A reduction in the growth rate of the organisms at all the three alkaline $\mathrm{pH}$ was observed. The growth curve of the isolated organisms at three different acidic $\mathrm{pH}$ is given in the Figure 2. There is reduction in the growth rate of the organisms at all the three acidic $\mathrm{pH}$ and the highest reduction was at a $\mathrm{pH}$ of 6.3 . The growth curve of the isolated organisms at three different temperature is given in the Figure 3. There is significant reduction in the growth rate of the organisms at all the temperature and the highest reduction was at $70^{\circ} \mathrm{C}$.

Fig. 1 Growth curve of acid adapted organisms

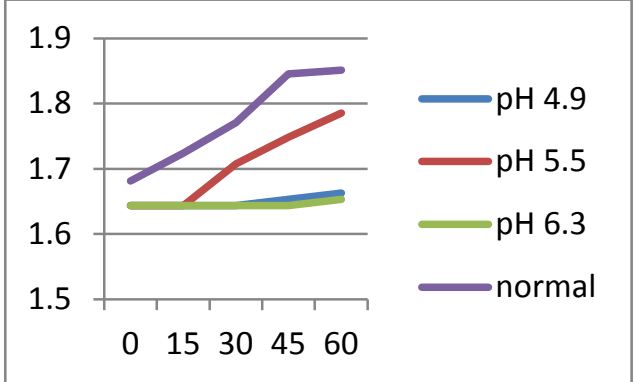

Fig. 3 Growth curve of heat adapted organisms

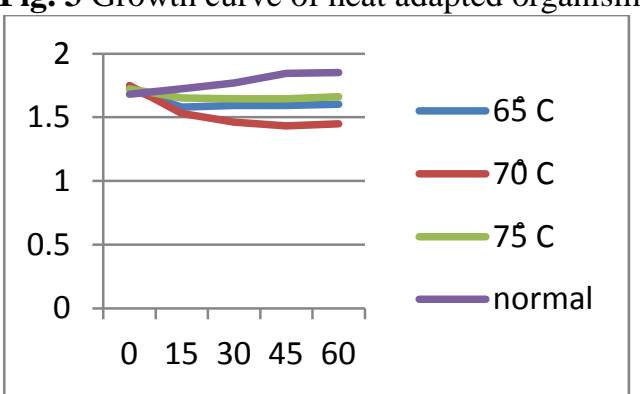

Fig. 2 Growth curve of alkali adapted organisms

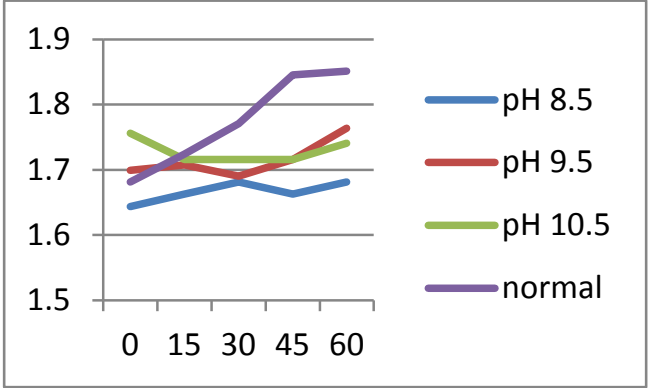

\section{Discussion}

The maximum growth was obtained at a pH of 5.5 followed by 4.9 and 6.3. This is similar to the findings of Lindsaya et al. (2000), in which the maximum growth was occurred at a $\mathrm{pH}$ of 5.8 for B pumilus and growth rate decreased form 5.9 to 7 . As the maximum inhibition to the growth rate was observed at a pH of 6.3 , the concentration of acidic solution for cleaning can be adjusted to this $\mathrm{pH}$ for more cleaning efficiency. The alkaline adaptation studies by enumeration shows that the growth of the organism was almost similar at $\mathrm{pH} 9.5$ and 10.5. However, at a $\mathrm{pH} 8.5$ the growth rate of organism was much more reduced. This is not in agreement with the findings of the Lindsaya et al. (2000), who reported a maximum growth rate at a $\mathrm{pH}$ of 7.8 followed by 8.9. In contrast to that in the present study at $\mathrm{pH} 8.5$ maximum inhibition of growth occurred and as $\mathrm{pH}$ increasing the growth rate is increased. The enumeration of the heat adaptation of the isolated organisms showed that the growth of the organisms was maximum inhibited at $70^{\circ} \mathrm{C}$ followed by $65^{\circ} \mathrm{C}$ and $75^{\circ} \mathrm{C}$.

\section{Conclusion}

For the maximum inhibition of the Bacilli which is predominant in the various samples of alkali cleaning solution tested, it is recommended to use minimum of $70^{\circ} \mathrm{C}$ for its destruction. The preferred $\mathrm{pH}$ for alkaline and acid cleaning solution were found to be 8.5 and 6.3 respectively.

\section{References}

[1]. Alvarez, S., N. Gobbato, E., Bru, A. P., Holagdo, D.R. and Perdigon, G. 1998. Specific immunity induction at the mucosal level by viable Lactobacillus casei, A perspective for oral vaccine development. Food Agri. Immunol. 10:79-87

[2]. Lindsaya, D., Brozelb, V. S., Mosterta, J. F. and Holya, A. V. 2000. Physiology of dairy associated Bacillus spp. over a wide pH range. Int. J. food microbiol. 54: 49-62

[3]. Mortan, D.R. 2001. Aerobic plate count. Compendium of methods for the microbial examination of foods. (eds. Downes, F.P. and Ito, K.). Fourth edition. American Public Health Association, Washington DC, pp. 63-68 